\title{
Modulation of Luttinger liquid exponents in multiwalled carbon nanotubes
}

\author{
S. Bellucci, ${ }^{1}$ J. González, ${ }^{2}$ P. Onorato,,${ }^{1,3}$ and E. Perfetto ${ }^{1,2}$ \\ ${ }^{1}$ INFN, Laboratori Nazionali di Frascati, P.O. Box 13, 00044 Frascati, Italy \\ ${ }^{2}$ Instituto de Estructura de la Materia, Consejo Superior de Investigaciones Cientficas, Serrano 123, 28006 Madrid, Spain \\ ${ }^{3}$ Dipartimento di Scienze Fisiche, Università di Roma Tre, Via della Vasca Navale 84, 00146 Roma, Italy
}

(Received 21 December 2005; published 26 July 2006)

\begin{abstract}
We develop in this paper a theoretical framework that applies to the intermediate regime between the Coulomb blockade and the Luttinger liquid behavior in multiwalled carbon nanotubes. Our main goal is to confront the experimental observations of transport properties, under conditions in which the thermal energy is comparable to the spacing between the single-particle levels. For this purpose we have devised a many-body approach to the one-dimensional electron system, incorporating the effects of a discrete spectrum. We show that, in the crossover regime, the tunneling conductance follows a power-law behavior as a function of the temperature, with an exponent that oscillates with the gate voltage as observed in the experiments. Also in agreement with the experimental observations, a distinctive feature of our approach is the existence of an inflection point in the log-log plots of the conductance vs temperature, at gate voltages corresponding to peaks in the oscillation of the exponent. Moreover, we evaluate the effects of a transverse magnetic field on the transport properties of the multiwalled nanotubes. For fields of the order of $4 \mathrm{~T}$, we find changes in the band structure that may be already significant for the outer shells, leading to an appreciable variation in the powerlaw behavior of the conductance. We then foresee the appearance of sizeable modulations in the exponent of the conductance for higher magnetic fields, as the different subbands are shifted towards the development of flat Landau levels.
\end{abstract}

DOI: $10.1103 /$ PhysRevB.74.045427

PACS number(s): 73.63.Fg, 73.22.-f, 73.23.-b

\section{INTRODUCTION}

Recent progresses in nanotechnology has revealed many interesting transport phenomena in mesoscopic lowdimensional structures. Carbon nanotubes (CNs) were discovered by Iijima in $1991,{ }^{1}$ as a by-product of carbon fullerene production. CNs are basically rolled up sheets of graphite (hexagonal networks of carbon atoms) forming tubes that are only nanometers in diameter. The electronic properties of $\mathrm{CNs}$ depend on their diameter and chiral angle (helicity) parametrized by a roll-up (wrapping) vector $(n, m){ }^{2}$ Hence it follows that some nanotubes are metallic with high electrical conductivity, while others are semiconducting with relatively low band gaps. They may also display different electronic properties depending on whether they are single-walled carbon nanotubes (SWNTs) or multiwalled carbon nanotubes (MWNTs). MWNTs are typically made of several (typically ten) concentrically arranged graphene sheets with a radius of about $5 \mathrm{~nm}$ and lengths in the range of $1-100 \mu \mathrm{m}$.

Several electrical transport experiments have been done by using CNs. In these experiments the effects of the Coulomb interaction are quite relevant, and the way they manifest themselves depends mainly on the size of the system, the temperature, and the quality of the contacts used in experiments. Thus MWNTs show for instance different transport regimes from ballistic transport (conductance quantization, 3,4 Luttinger-liquid-like ${ }^{5,6}$ ) to diffusive transport $^{7}$ and also strong localization. ${ }^{8,9}$

When the contacts are not highly transparent, the measurements of the conductance and the differential conductivity reflect the strong Coulomb repulsion in CNs. ${ }^{10,11}$ At low temperature, $T \leqq 1 \mathrm{~K}$, the zero-bias conductance exhibits regular oscillations corresponding to the so-called single electron tunneling (SET), ${ }^{12,13}$ or Coulomb blockade (CB). The addition energy ${ }^{12}$ depends on the level spacing, due to the momentum quantization, and on the electron-electron interaction. ${ }^{14}$ The differential conductivity of individual MWNTs has been measured in Ref. 15, where CB and energy-level quantization have been observed.

When the thermal energy is much larger than the level spacing, the transport measurements reflect instead the many-body properties of the system. In fact, the electronelectron interaction in a one-dimensional (1D) system is expected to lead to the formation of a Luttinger liquid (LL) with unusual electronic properties that cannot be explained by Fermi liquid theory. ${ }^{16-19}$

Characteristic experimental signatures support the assumed LL behavior of $\mathrm{CNs}^{20-23}$ In fact the tunneling conductance $G$ reflects the power-law dependence of the TDOS in a small-bias experiment, ${ }^{24}$

$$
G=d I / d V \propto T^{\alpha},
$$

for $e V_{b} \ll k_{B} T, V_{b}$ being the bias voltage. Such kind of signatures of the LL behavior have been observed in single-walled as well as in multiwalled nanotubes. In MWNTs the observed values of $\alpha$ lie between 0.04 and 0.3 , and results in agreement with the experimental values have been obtained in previous theoretical works. ${ }^{25-28}$

In this paper we introduce a theoretical description of the crossover as the temperature decreases from the LL regime to the $\mathrm{CB}$ regime. We do so in order to confront the experimental data reported in a recent letter by Kanda et al. ${ }^{29}$ in which the intermediate regime has been explored measuring the zero-bias conductance at temperatures where the thermal energy becomes comparable to the level spacing in the dis- 
crete single-particle spectrum. In Ref. 29 the authors have reported a systematic study of the gate voltage dependence of the LL-like behavior in MWNTs, showing periodic oscillations of the exponent $\alpha$ on the gate voltage $V_{g}$, with values of $\alpha$ ranging from 0.05 to 0.35 . Changes in the exponent $\alpha$ are observed in the plots of the conductance at an inflection temperature $T^{*} \sim 30 \mathrm{~K}$, for values of $V_{g}$ corresponding to peaks of $\alpha$. Remarkably the exponent $\alpha$ depends significantly on the transverse magnetic field acting on the CN. In particular $\alpha$ is reduced from 0.34 , at a peak in the $\alpha$ oscillations, to 0.11 , for a magnetic field $B \sim 4 \mathrm{~T}$.

In their paper, Kanda et al. do not find it plausible to explain the mentioned features starting from a LL description of MWNTs. In fact they discussed two different theories, the first one being based on a model for MWNTs composed of several ballistic metallic shells ${ }^{30}$ and the second one, known as nonconventional Coulomb blockade (NCCB), considering the MWNT as a diffusive conductor. ${ }^{31}$ Kanda and coworkers conclude that the latter theory explains better the experimental results, by assuming that the mean-free path $l$ may fluctuate with the gate voltage, according to the theoretical predictions by Choi et al. ${ }^{32}$ that have studied the effects of single defects on the local density of states via resonant backscattering. We believe otherwise, that the depedence of the $\alpha$ exponent on the gate voltage is in correspondence with a definite periodic structure of the single-particle density of states. ${ }^{33}$

Thus in this paper we discuss in more detail some crucial aspects of the approach presented in Ref. 33, also by giving more accurate results, and we extend our theory to the effects of a transverse magnetic field, by giving some correspondences between our theory and the experiment.

In the next section, we put forward the idea that there exists a clear relation between the periodic oscillations in the $\alpha$ exponent and the quantization of the energy levels in MWNTs. ${ }^{33}$ Building up on this idea, we introduce an energy distribution function which takes into account the effects of the quantization at low temperature and can be compared with the Fermi-Dirac distribution in the high-temperature limit. Next, in Sec. III we develop a theoretical approach based on many-body theory, in order to deal with the lowenergy effects of the long-range Coulomb interaction in the 1D electron system. We introduce in that scheme the discussed energy distribution function, for the sake of evaluating the effects of the discrete spectrum on the exponent $\alpha$. The results of this approach are reported in Sec. IV, where we carry out the comparison between theoretical predictions and experiments. The effects of a transverse magnetic field are considered in Sec. V. Then, in Sec. VI, we highlight how the distinctive features of our approach match naturally with experimental observations.

\section{SINGLE-PARTICLE PROPERTIES}

The experimental data of Ref. 29 suggest a relation between the period $\Delta V_{g}$ of the oscillations in the $\alpha$ exponent and the quantization of energy levels in MWNTs, ${ }^{33} \Delta \varepsilon$ $\approx v_{F} h / L$, where $L$ is the length of the sample (i.e., $\Delta V_{g}$ $\propto \Delta \varepsilon$ ). This supports a theory based on a microscopic de- scription of the single-particle spectrum, in order to explain the oscillations of $\alpha$. Here the quantized energy levels play a central role. This theory has to hold in the intermediate regime between very low temperature, where the peaks of the CB are measured by increasing $V_{g}$, and room temperature, where the LL behavior prevails. To this aim, we will rely on the 1D many-body approach, incorporating at the same time the discrete character of the spectrum which arises from the finite geometry of the system.

In the introduction of microscopic features in the manybody formalism, a central role is played by the energy distribution function (EDF) $n\left(\varepsilon ; \varepsilon_{F}\right)$. In the many-body approach to electron liquids, the function $n\left(\varepsilon, \varepsilon_{F}\right)$ corresponds to the Fermi-Dirac distribution, which is usually replaced by the Heaviside step function $\vartheta\left(\varepsilon-\varepsilon_{F}\right)$ in order to simplify the calculations.

At very low temperatures, as those typical for the $\mathrm{CB}$ regime, the EDF has to reflect the discreteness of energy levels. Thus some pattern of peaks, corresponding to quantized energy levels, has to be present. Hence at low temperatures, where the microscopic level quantization is manifest, peaks arise with a shape made smooth by the growth of the temperature. The spacing of the peaks is proportional to $\Delta \varepsilon$. The latter value yields a temperature $T_{c} \sim \Delta \varepsilon / k_{B}$, above which we expect that the oscillations will be smoothed.

The thermal energy $k_{B} T_{c}$ governs then the crossover between two different regimes. From our analysis, it is clear that two scales for the behavior in temperature characterize the system, corresponding to longitudinal and transverse quantization energies, although the second one turns out to be about 10-100 times larger than $k_{B} T_{c}$.

We can model the $n$th peak of the EDF by a normalized function $f(\varepsilon, n)$, with a dependence on temperature consistent with the line shape for a thermally broadened resonance, ${ }^{34}$

$$
f(\varepsilon, n)=\frac{1}{4 k T \cosh ^{2}\left(\frac{\varepsilon-n \Delta \varepsilon}{2 k T}\right)},
$$

where $n \Delta \varepsilon$ stands for the position of the $n$th energy level. The energy distribution function is cutoff by the Fermi en$\operatorname{ergy} \varepsilon_{F}$, as it is given by

$$
n\left(\varepsilon ; \varepsilon_{F}\right)=\sum_{n=0}^{\infty} f(\varepsilon, n) \theta\left(\varepsilon_{F}-\varepsilon\right) .
$$

Alternatively we could introduce a smooth cutoff at the Fermi energy, taking into account thermal effects,

$$
n\left(\varepsilon ; \varepsilon_{F}\right)=\sum_{n=0}^{\infty} f(\varepsilon, n) \frac{1}{1+e^{\left(\varepsilon-\varepsilon_{F}\right) / k T}} .
$$

However, the sharp cutoff given by $\theta\left(\varepsilon_{F}-\varepsilon\right)$ is more convenient, in order to proceed with analytical computations. On the other hand, we have checked that the presence of the smooth cutoff does not change appreciably the whole scenario.

At high temperatures, we can neglect the level spacing and calculate the EDF by carrying out the integral 


$$
n\left(\varepsilon ; \varepsilon_{F}\right) \approx \int_{-\infty}^{\varepsilon_{F} / \Delta \varepsilon-1 / 2} d y f(\varepsilon, y)=\frac{1}{1+e^{\left(\varepsilon-\varepsilon_{F}+\Delta \varepsilon / 2\right) / k T}} .
$$

The latter formula gives the Fermi-Dirac distribution, which is usually approximated with a step function. However, the energy distribution function has in general a more structured shape, that depends on the ratio between the level spacing $\Delta \varepsilon$ and the thermal energy $k T$, as illustrated in Fig. 1 of Ref. 33.

The structure of peaks in the energy distribution function affects several objects in the many-body theory, such as the one-loop polarization $\Pi^{(0)}\left(q, \omega_{q}\right)$. This function provides us a counting for the number of particle-hole excitations that can be built with momentum $q$ across the Fermi level. Thus in the case of a model with linear dispersion $\varepsilon(k) \approx v_{F} k$, it becomes proportional at zero temperature to the integral

$$
\int_{-\infty}^{\infty} \frac{d k}{2 \pi} \frac{\theta\left(q+k-k_{F}\right)-\theta\left(k-k_{F}\right)}{v_{F} q}=\frac{1}{v_{F} q} \int_{0}^{q} \frac{d k}{2 \pi}=\frac{1}{2 \pi v_{F}} .
$$

In the approach based on the distribution function given by Eq. (3), the peaks give rise to a factor in the integration that reflects the discrete structure of the system. Therefore Eq. (6) becomes

$$
\begin{aligned}
& \int_{-\infty}^{\infty} \frac{d k}{2 \pi} \Delta \varepsilon \frac{n\left(v_{F}(q+k) ; \varepsilon_{F}\right)-n\left(v_{F} k ; \varepsilon_{F}\right)}{v_{F} q} \\
& =\frac{1}{v_{F} q} \int_{0}^{q} \frac{d k}{2 \pi} \Delta \varepsilon \sum_{n=0}^{\infty} f\left(v_{F} k, n\right) \approx \frac{1}{2 \pi v_{F}} N_{T}\left(\varepsilon_{F}\right),
\end{aligned}
$$

where, to the first order, the function $N_{T}\left(\varepsilon_{F}\right)$ can be taken to be independent on $q$. Considering the sharp cutoff, the $N_{T}$ function gets a simple form

$$
\begin{aligned}
\int_{0}^{q} d k \Delta \varepsilon \sum_{n=0}^{\infty} f\left(v_{F} k, n\right) \\
\quad=\frac{\Delta \varepsilon}{2 v_{F}} \sum_{n=0}^{\infty}\left[\tanh \left(\frac{\varepsilon_{F}+v_{F} q-n \Delta \varepsilon}{2 k T}\right)-\tanh \left(\frac{\varepsilon_{F}-n \Delta \varepsilon}{2 k T}\right)\right] \\
\quad \approx q \frac{\Delta \varepsilon}{4 k T} \sum_{n=0}^{\infty}\left[1-\tanh ^{2}\left(\frac{\varepsilon_{F}-n \Delta \varepsilon}{2 k T}\right)\right]=q N_{T}\left(\varepsilon_{F}\right)
\end{aligned}
$$

Thus it holds that

$$
N_{T}\left(\varepsilon_{F}\right)=\frac{\Delta \varepsilon}{4 k T} \sum_{n=0}^{\infty}\left[1-\tanh ^{2}\left(\frac{\varepsilon_{F}-n \Delta \varepsilon}{2 k T}\right)\right] .
$$

This function is plotted in Fig. 1. As expected, $N_{T}(\varepsilon)$ is periodic with period $\Delta \varepsilon$.

We introduced the function $N_{T}$ depending on the Fermi energy $\varepsilon_{F}$. In this respect, the position of $\varepsilon_{F}$, tunable by the gate voltage $V_{g}$, fixes the portion of the last peak that we have to integrate. It is clear that, from an experimental point of view, $\varepsilon_{F}$ is in correspondence with the value of the gate

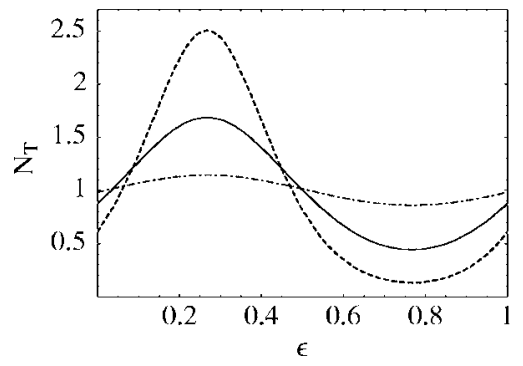

FIG. 1. Plot of $N_{T}\left(\varepsilon_{F}\right)$ for three different values of $k T / \Delta \varepsilon$ : $k T / \Delta \varepsilon=0.1$ (dashed), $=0.15$ (solid), $=0.3$ (dotted-dashed). The value of $\varepsilon$ is given in units of $\Delta \varepsilon$

voltage $V_{g}$, so that it can be shifted in a continuous way, allowing for the exploration of the region between two peaks in Fig. 1 of Ref. 33.

\section{MANY-BODY APPROACH}

In CNs, the relevant interaction is given by the large Coulomb potential at small momentum transfer. ${ }^{35,36}$ It is known that the Coulomb interaction remains long ranged in a $1 D$ electron system, ${ }^{25,37}$ and this property is also shared by the graphene sheet, which has a vanishing 2D density of states at the Fermi level. In order to obtain a sensible expression of the 1D interaction, we may start from the representation of the $1 /|\mathbf{r}|$ Coulomb potential in three spatial dimensions, as the Fourier transform of the propagator $1 / \mathbf{k}^{2}$,

$$
\frac{1}{|\mathbf{r}|}=\int \frac{d^{3} k}{(2 \pi)^{3}} e^{i \mathbf{k} \cdot \mathbf{r}} \frac{1}{\mathbf{k}^{2}} \text {. }
$$

If the interaction is projected onto one spatial dimension, by integrating for instance the modes in the transverse dimensions, then the Fourier transform has a logarithmic dependence on the longitudinal momentum. ${ }^{25}$ In our case, the scale of the longitudinal momentum $k$ in the logarithm is dictated by the existence of a short-distance cutoff $k_{c}$, stemming from the small transverse size of the electron system $\left(k_{c} \sim 1 / R\right)$. Thus we end up with a representation of the Fourier transform $\tilde{V}(k)$ of the 1D Coulomb potential,

$$
\tilde{V}(k) \approx 1 /(2 \pi) \ln \left(k_{c} / k\right) .
$$

Our interest is focused on the description of the interaction among a number $N_{s}$ of subbands crossing the Fermi level in the conducting shell of a multiwalled nanotube.

Thus we follow Refs. 25-28, where we neglect interactions that lead to a change of chirality, as they imply a large momentum transfer of the order of $2 k_{F}$ and are therefore subdominant, with respect to the large Coulomb interaction at small momentum transfer. Such so-called backscattering interactions are known to remain negligible down to extremely low energies. On the other hand, the model built from interactions between currents with a well-defined chirality has some interesting properties. One of them is that, when performing the perturbative calculation of the polarization function $\Pi\left(q, \omega_{q}\right)$, the self-energy corrections cancel order by order against the vertex corrections. Thus the exact 


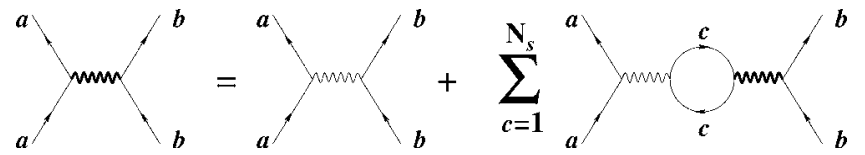

FIG. 2. Self-consistent diagrammatic equation for the dressed Coulomb interaction.

result is just given by the bare polarization function. In our case, upon the introduction of the different subbands and the mentioned effects in the single-particle density of states, we have

$$
\Pi\left(q, \omega_{q}\right)=\frac{2}{\pi} N_{s} N_{T}\left(\varepsilon_{F}\right) \frac{v_{F} q^{2}}{\omega_{q}^{2}-v_{F}^{2} q^{2}} .
$$

Moreover, there is further simplification of the diagrammatics coming from the fact that all fermion loops with more than two interaction lines vanish identically in onedimension. Thus the dressed Coulomb interaction has to satisfy the self-consistent equation shown in Fig. 2, that amounts to the RPA sum of particle-hole contributions.

As long as the RPA provides the exact expression of the dressed Coulomb interaction, a sensible representation of the electron self-energy $\Sigma\left(q, \omega_{q}\right)$ is given by the partial sum of the perturbative expansion shown in Fig. 3. Correspondingly, we may express the electron self-energy in the form

$$
\begin{aligned}
\Sigma\left(k, \omega_{k}\right)= & i \int_{-k_{c}}^{k_{c}} \frac{d q}{2 \pi} \int_{-\infty}^{\infty} \frac{d \omega_{q}}{2 \pi} G^{(0)}\left(k-q, \omega_{k}-\omega_{q}\right) \\
& \times \frac{\widetilde{V}(q)}{1-\tilde{V}(q) \Pi\left(q, \omega_{q}\right)},
\end{aligned}
$$

where $G^{(0)}\left(k, \omega_{k}\right)$ stands for the free electron propagator. For the evaluation of the scaling properties of the quasiparticle parameters, it is enough to obtain the logarithmic dependence of the function $\Sigma\left(q, \omega_{q}\right)$ on the high-energy cutoff $E_{c}$ $\sim v_{F} k_{c}$. Thus the representation in Fig. 3 misses corrections to the fermion line $G^{(0)}$, as well as corrections to the threepoint vertex at the end of the line, but they do not introduce further divergences in the cutoff $E_{c}$ and can be safely disregarded in the determination of the electron field scale.

We therefore concentrate ourselves on the computation of the contributions that diverge with the cutoff $E_{c}$ in Eq. (13). Order by order in perturbation theory, we find that the same kind of logarithmic dependence appears in linear terms proportional to $k$ and $\omega_{k}$. We can recast the whole perturbative series $^{25-28}$ into the expression

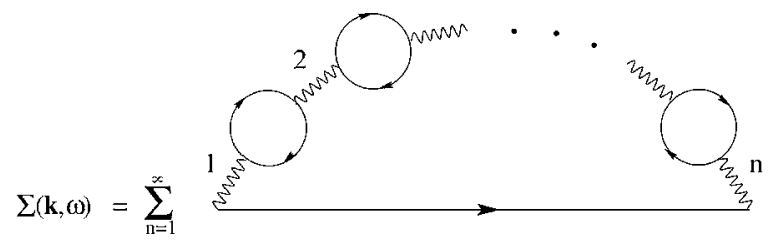

FIG. 3. Set of diagrams for the computation of the electron self-energy, where each particle-hole bubble represents the full polarization operator.

$$
\Sigma\left(k, \omega_{k}\right) \approx \alpha(g)\left(\omega_{k} \pm v_{F} k\right) \ln \left(E_{c}\right)
$$

where the effective coupling is given by $g$ $=2 N_{s} N_{T} \tilde{V}\left(q_{0}\right) e^{2} / \pi v_{F}$, in terms of a suitable average momentum $q_{0}{ }^{38}$

Following the calculation reported in Refs. $25-28$ we get the scaling equation for the $Z$ factor,

$$
E_{c} \frac{d}{d E_{c}} \ln Z\left(E_{c}\right)=\alpha(g),
$$

where

$$
\alpha(g)=\frac{1}{4 N_{s} N_{T}}\left(\sqrt{1+g}+\frac{1}{\sqrt{1+g}}-2\right) .
$$

We observe that $Z\left(E_{c}\right)$ follows a power-law behavior as a function of the energy cutoff $E_{c}$. We may identify the factor $Z\left(E_{c}\right)$ with the weight of the electron quasiparticles, which vanishes as the Fermi level is approached in the limit $E_{c}$ $\rightarrow 0$. We capture, in this way, one of the most genuine features of the many-body theory in one dimension, namely the absence of low-energy excitations with Fermionic character, which defines the Luttinger liquid class of $1 \mathrm{D}$ electron liquids.

\section{TUNNELING DENSITY OF STATES}

The power-law behavior of the quasiparticle weight $Z\left(E_{c}\right)$ translates into a similar dependence on energy of the tunneling density of states $n(\varepsilon)$. By trading the dependence on the high-energy cutoff with the energy measured with respect to the Fermi level, we obtain the scaling behavior

$$
n(\varepsilon) \sim N_{s} Z(\varepsilon) \sim \varepsilon^{\alpha(g)} .
$$

Usually the behavior of the TDOS is obtained by measuring its dependence on temperature or bias voltage. The exponent in Eq. (17) has then to be compared with the measurements of a power-law behavior observed in the plots of the conductance versus temperature, or in the differential conductivity.

Regarding the experiments in Ref. 29, we are in the position to explain the oscillations observed in the exponent of the conductance, in a temperature regime corresponding to a thermal energy of the order of the level spacing $\Delta \varepsilon$. The point is that, as we shift the Fermi level along the structure of single-particle peaks in the spectrum, the $\alpha$ exponent has to reflect the modulation of the periodic factor $N_{T}(\varepsilon)$. As mentioned before, the period of oscillations is in agreement with the change in the gate voltage required, for a shift $\Delta \varepsilon$ of the Fermi level. Next, we comment upon several features regarding the amplitude of the oscillations.

It is very convenient to plot the expression of the exponent $\alpha(g)$ as a function of the variable $N_{S} N_{T}$. This depends also on the effective coupling $\widetilde{V}\left(q_{0}\right) e^{2} / v_{F}$ of the Coulomb interaction. We have estimated this quantity by using a value of $q_{0}$ consistent with the nanotube length, and taking into account the screening of the interaction, mainly by other shells in the nanotube. Thus sensible values of $\widetilde{V}\left(q_{0}\right) e^{2} / v_{F}$ turn out to be of the order of $\sim 10$. The exponent $\alpha$ has been 


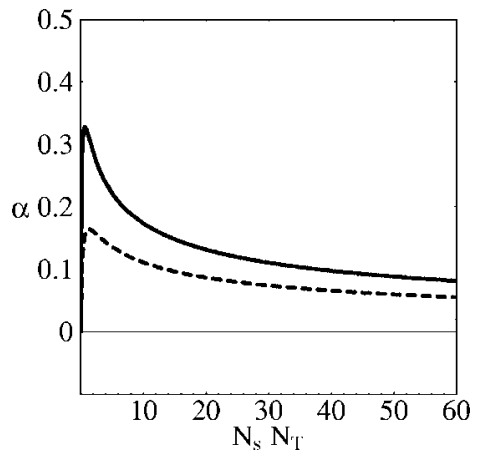

FIG. 4. Plot of the exponent $\alpha$ as a function of the product of the number of subbands $N_{s}$ and the weight $N_{T}$ in the single-particle spectrum.

represented in Fig. 4 as a function of $N_{s} N_{T}$, for two different choices of the effective coupling. We may assume the typical situation in which the MWNTs are hole doped, with a number of subbands crossing the Fermi level $N_{s} \approx 6-10 .{ }^{39} \mathrm{We}$ observe that, for such an estimate of $N_{s}$, the modulation of the $N_{T}$ factor (with the amplitude reported in Sec. III) accounts for oscillations of $\alpha$ reaching values as high as $\approx 0.3$ (for a large value of the effective coupling) and as low as $\approx 0.05$ (for a small value of $g$ and a large number of subbands).

It is actually quite plausible that the drift observed, in general, in the average value of the $\alpha$ exponent (i.e., towards larger values, for higher gate voltages) may be the consequence of a change in the Fermi velocity, as the Fermi level approaches the top of the lowest partially filled subband. We note that the height reached by the oscillations in the $\alpha$ exponent is different in the two plots of experimental measurements shown in Figs. 2(c) and 2(d) in Ref. 29. This may be attributed to a different relative position of the Fermi level between two consecutive subbands in the nanotube band structure. We have modeled the proximity to the point with divergent density of states, at the top $\varepsilon_{T}$ of a given subband, by allowing for a dependence of the Fermi velocity $v_{F}\left(\varepsilon_{F}\right)$ $\sim \sqrt{\varepsilon_{T}-\varepsilon_{F}}$. When this is taken into account, in conjunction with the modulation of the periodic function $N_{T}\left(\varepsilon_{F}\right)$, we obtain a picture for the dependence of the $\alpha$ exponent on the gate voltage which is in good qualitative agreement with the oscillations reported in Ref. 29, as shown in Fig. 2 of Ref. 33.

We observe the similarity of the plot in Fig. 2 of Ref. 33 with the experimental curves in Figs. 2(c) and 2(d) of Ref. 29 for the exponent, over a $20-\mathrm{V}$ range in the gate voltage. The oscillations are significant for low values of the thermal energy $k_{B} T$, as compared to $\Delta \varepsilon$. For higher temperatures the thermal fluctuations erase the structure of superposed peaks in Eq. (3), which leads in turn to the disappearance of the oscillations in the $\alpha$ exponent.

An important point reinforcing the above interpretation of the experimental measurements is that the depedendence on the variable $N_{s} N_{T}\left(\varepsilon_{F}\right)$ shown in Fig. 4 translates naturally into an asymmetric behavior of the exponent $\alpha$, when the oscillations approach the peak of the curve near $\alpha \approx 0.3$. If we consider, for instance, a doped nanotube with $N_{s}=10$ and an effective coupling $\widetilde{V}\left(q_{0}\right) e^{2} / v_{F} \approx 12$, we observe that the exponent may run from a value $\alpha \approx 0.18$, for $N_{T}=1$, to a value $\alpha \approx 0.3$, for a reasonably small value of $N_{T}$. However, it is clear that the other side of the oscillation does not have the same magnitude. This is consistent with the fact that inflection points in the plots of the conductance versus temperature have been observed for peak values in the exponent oscillations. In our model, the values corresponding to a dip for $N_{T}>1$ do not deviate much from the high-temperature behavior recovered for $N_{T} \approx 1$. It is also remarkable that the inflection points have been found experimentally to correspond to the highest oscillations, for a large gate voltage. Once again, this is consistent with our theoretical interpretation, in which the lower values of the exponent $\alpha$ correspond to smaller values of the effective coupling, that do not support large variations upon changes in $N_{T}$, as shown from the lower curve in Fig. 4.

\section{MAGNETIC-FIELD EFFECTS}

The experiments reported in Ref. 29 have also shown that the exponent in the power-law behavior of the zero-bias conductance may depend on the applied transverse magnetic field. The magnetic length $l=\sqrt{c / e B}$ corresponding to the field strength used in the experiments $(B=4 \mathrm{~T})$ is anyhow larger than the radius of the outer shells in MWNTs. Then, the resulting band structure must be far from the development of Landau levels, although it must already lead to the features producing the variation observed in the $\alpha$ exponent.

In order to study the influence of the magnetic field, we analyze the change that it may produce in the band structure of nanotubes with different chiralities. Previous investigations have addressed the formation of Landau subbands in carbon nanotubes upon application of a transverse magnetic field. Here we have undertaken a similar computational approach, but applied to nanotubes of large radius $R \sim 10 \mathrm{~nm}$, corresponding to active shells in multiwalled nanotubes. We have thus resorted to a tight-binding calculation of the Hamiltonian in the carbon lattice, with the usual prescription of correcting the transfer integral by appropriate phase factors

$$
\exp \left(i \frac{e}{c} \int_{\mathbf{r}}^{\mathbf{r}^{\prime}} \mathbf{A} \cdot \mathbf{d l}\right)
$$

depending on the vector potential $\mathbf{A}$ between nearestneighboring sites $\mathbf{r}$ and $\mathbf{r}^{\prime}$. The vector potential has been chosen, in particular, with the appropriate functional dependence at the nanotube surface, as described in Ref. 40.

The results of the numerical diagonalization of the tightbinding Hamiltonian for a magnetic field $B=4 \mathrm{~T}$ are shown in Fig. 5. The band structure corresponds to the particular case of a zigzag nanotube, but it is quite representative of the changes produced in chiral nanotubes upon application of the magnetic field. There is an incipient tendency of the subbands to flatten out, an effect which is a precursor of the formation of Landau subbands at much higher values of the magnetic field. The two low-energy subbands spanning the gap for semiconducting nanotubes tend to approach each 


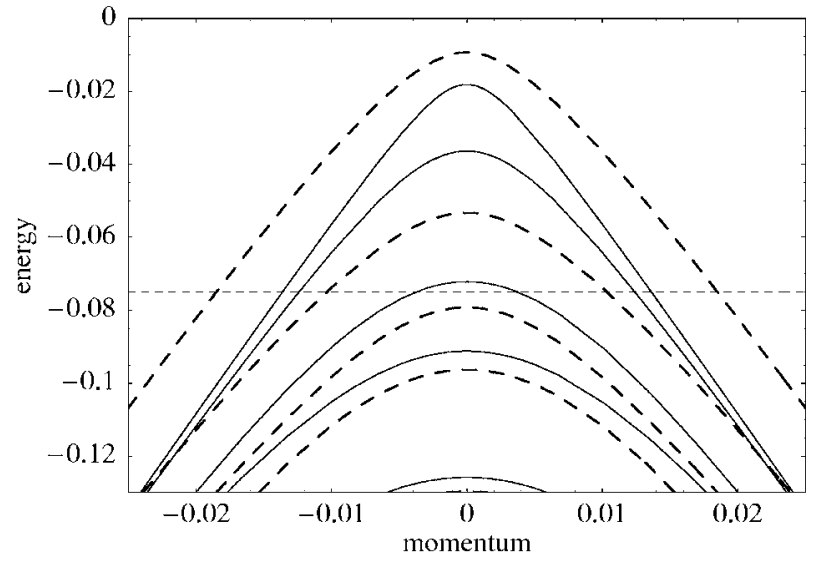

FIG. 5. Energy dispersion relation of a $(280,0)$ zigzag nanotube with $B=0$ (solid lines) and $B=4 \mathrm{~T}$ (dashed lines). The position of the Fermi level for a hole-doped nanotube is also indicated. The energy appears in units of $\mathrm{eV}$ (we used a hopping parameter $t=2.5 \mathrm{eV}$ between nearest-neighbor carbon atoms) and the momentum is given in $\AA^{-1}$ ).

other, as the lowest Landau level corresponds always to zero energy in carbon nanotubes. The next subbands move in the opposite direction, towards a very slow rearranging along the direction of higher Landau levels.

We also calculated the effect of the perpendicular magnetic field by means of an alternative approach based on a model of massless Dirac electrons on a cylinder. ${ }^{41}$ Such an approach is very accurate for thick nanotubes and magnetic fields of moderate intensity ${ }^{41}$ and it provides results in good agreement with the tight-binding calculation. In Fig. 6 we show the modification in the band structure for a metallic, e.g., armchair, $\mathrm{CN}$ obtained by the numerical solution of the Dirac-like Hamiltonian in the presence of a magnetic field. ${ }^{41}$

In both cases there is a significant modification in the low-energy spectrum whenever the number of subbands crossing the Fermi level changes, as a consequence of switching on the magnetic field. This happens, in particular, when the Fermi level for $B=0$ is slightly below the top of one of the subbands (see Fig. 5), which is pushed downwards under the action of the magnetic field. Recalling the discus-

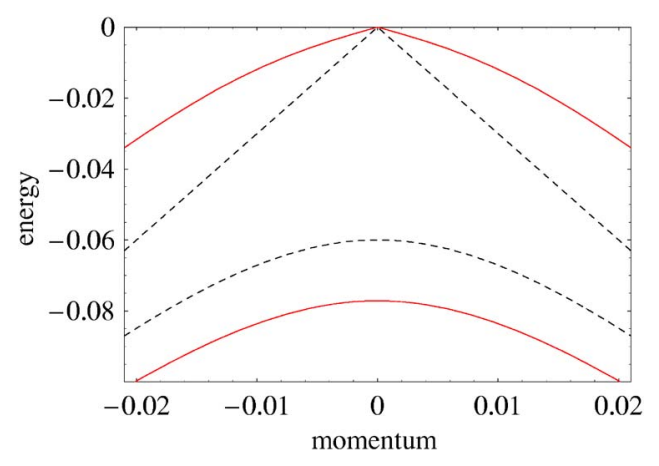

FIG. 6. (Color online) Energy dispersion relation of a metallic, e.g., armchair, nanotube with radius $\sim 10 \mathrm{~nm}$, for $B=0$ (solid lines) and $B=4 \mathrm{~T}$ (dashed lines). The energy appears in units of eV and the momentum is given in $\AA^{-1}$ and is relative to one of the two Fermi points.

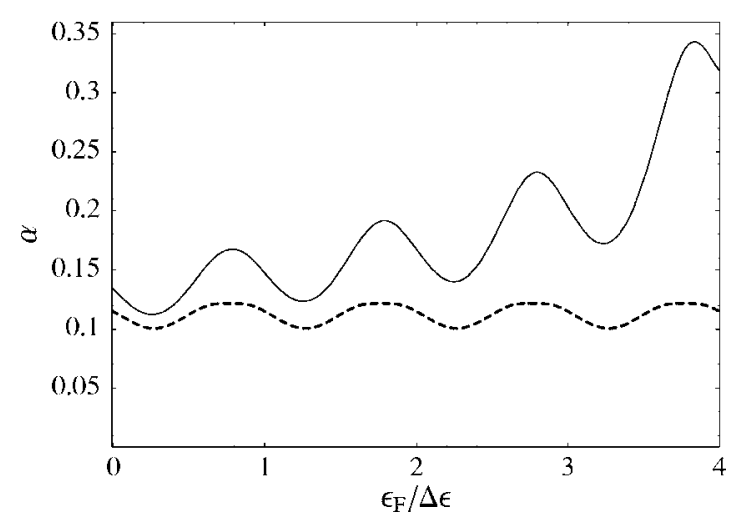

FIG. 7. Plot of the exponent $\alpha$ as a function of the Fermi energy $\varepsilon_{F}$ (in units of $\Delta \varepsilon$ ) for $B=0$ (solid line) and $B=4 \mathrm{~T}$ (dashed line). Here the temperature is $k T / \Delta \varepsilon=0.15$. The system is the same as in Fig. 5.

sion of the preceding section, this is the instance that corresponds to higher gate voltages in Fig. 2 of Ref. 33, where the value of the exponent $\alpha \approx 0.3$ is approached. Under these circumstances, a couple of degenerate subbands with large density of states are lost at the Fermi level, upon application of the magnetic field. Although the number $N_{s}$ of subbands contributing to the conduction decreases, the main effect influencing the computation of the exponent $\alpha$ comes from the larger value of $v_{F}$ in the remaining (outer) degenerate subbands. Taking a value of the Fermi velocity corresponding to the Fermi level shown in Fig. 5, we find that the exponent is reduced down to a value $\alpha \approx 0.1$, in agreement with the experimental observation reported in Ref. 29.

Although no measurements have been reported over a given range of the gate voltage in the presence of the magnetic field, it is very instructive to apply Eq. (16), to find the results corresponding to the change in the band structure shown in Fig. 5. The gate-voltage dependence obtained in this way for the $\alpha$ exponent at $B=4 \mathrm{~T}$ is represented in Fig. 7. We observe that the plot of the exponent is now below the curve corresponding to the oscillations at $B=0$, which is in agreement with the general statement made in Ref. 29. However, the comparison with the experimental observation reported at a dip in the oscillations $(\alpha \approx 0.06$ at zero magnetic field) shows that our prediction falls short of accounting for the observed reduction. The experimental result reported at $B=4 \mathrm{~T}, \alpha \approx 0.005$, implies anyhow too large an effect on transport properties, which is difficult to reconcile with the change in the band structure, for such a relatively small magnetic field. In this respect, we point out that the evidence for a well-defined continuous plot is not so clear, for that particular measurement of the conductance, as in other cases. More experimental input will be needed, over a complete range of gate voltages, in order to test the theoretical predictions, incorporating a more precise picture of the magneticfield effects on the transport properties. ${ }^{42}$

\section{CONCLUDING REMARKS}

In this paper we have developed a framework that applies to an intermediate regime between that of the Coulomb 
blockade and the Luttinger liquid behavior. In our opinion this represents a significant progress, with respect to the arguments presented in Ref. 29 to interpret the experimental observations. There, it was actually claimed that a nonconventional Coulomb blockade (NCCB), arising from tunneling into a strongly interacting disordered metal, could originate the exponent oscillations. In fact, the NCCB predicts a power-law behavior of the conductance, with an exponent given in terms of the mean free path $l$ by $\alpha \approx R / N_{s} l$. Within this theory, the oscillatory behavior of the exponent was related to the possible modulation of the mean free path coming from disorder. This was supported by recalling the theoretical work of Choi et al..$^{32}$ about the influence of different forms of disorder on the local density of states. A closer look at this study shows, however, that it is highly unlikely that the combined effect of different defects may produce the desired oscillatory pattern.

From the results of Choi et al. in Ref. 32, we note that most of the impurities and defects of the carbon lattice have an effect on the density of states, extending over an energy range much larger than $1 \mathrm{meV}$. The latter yields an energy scale consistent with the observed oscillations in the $\alpha$ exponent. Thus from such a source of disorder we can only expect a more or less uniform effect on the range of gate voltage values shown in the plots of Figs. 2 (c) and 2(d) of Ref. 29. On the other hand, the effect of vacancies in the lattice produces much narrower peaks in the density of states, which now may give rise to features discernible at the meV scale. We believe that this may be the main origin of the irregularities observed in the oscillations of the $\alpha$ exponent.

Now we discuss the main distinctive features in the zerobias conductance of our theoretical approach, which lead to a very good agreement with the experimental observations reported in Ref. 29.

Our framework predicts the periodic dependence of the exponent $\alpha$ on the Fermi energy of the system, due to the oscillatory behavior of the factor $N_{T}\left(\varepsilon_{F}\right)$, as the Fermi level sweeps the structure of single-particle levels. As we have shown in Fig. 2 of Ref. 33, our model can explain the oscillations in the $\alpha$ exponent, leading to plots that are in good qualitative agreement with the experimental results, showing a dependence on the gate voltage and a period of oscillations consistent with the measured ones. In fact, the precise form of these experimental observations may change from one sample to another, but it remains clear that the oscillations with periodicity $\Delta V_{g}$ are in correspondence with the level spacing due to the quantization of single-particle levels.

In a recent experiment Kanbara et al. ${ }^{44}$ also investigated the gate voltage dependent conductance of a MWNT. In this case it was observed that $\alpha$ vanishes for high values of $V_{g}$. This can be easily explained in terms of the model we propose here. In fact, as we showed in our previous papers, ${ }^{27,28}$ and it is also clear from Eq. (16), the exponent $\alpha$ strongly decreases when increasing the number of channels that participate in the electron conduction, i.e., the number of subbands crossing the Fermi level. In Ref. 44 the authors calculated that $N_{s}$ goes from 1 to 40 in a range of $V_{g}$ between 0 and $20 \mathrm{~V}$. Thus the experimental setting is quite different from the one of Ref. 29, where the number of transverse modes is nearly constant.
Oscillations in the experimental plot of $\alpha$ vs $V_{g}$ were observed for values of $k_{B} T$ lower than a threshold, which is consistent with our estimate for the level spacing $\Delta \varepsilon$. Furthermore, our approach explains naturally the existence of an inflection point $T^{*}$ in the log-log plot of the conductance versus the temperature, for values of $V_{g}$ corresponding to peaks in the plot of $\alpha$. As reported in Ref. 29, the conductance keeps following an approximate power-law behavior below the temperature $T^{*}$, but with a critical exponent significantly enhanced, with respect to the high-temperature one. In our model, this is a natural consequence of the asymmetry in the range of variation of $\alpha$ in the upper plot of Fig. 4 , when the mean value of the product $N_{s} N_{T}$ is in the range $6-10$. Peak values of $\alpha$ as large as $\approx 0.3$ can be reached at low temperatures (which means $N_{T}<1$ ). However, minimum values of $\alpha$ are reached at the other side of the oscillation, for $N_{T}>1$. In that case, low temperature does not imply a significant change of the exponent with respect to the mean, high-temperature value. Thus our approach provides a consistent and comprehensive description of both the power-law dependence and the inflection point in the plot of the conductance as a function of temperature, as shown in Fig. 3 of Ref. 33.

The inflection point is more pronounced for gate voltages corresponding to larger values of $\alpha$, as observed in Ref. 29. Consistently, in our approach, smaller values of $\alpha$ correspond in general to oscillations along the lower curve in Fig. 4, which naturally yields less sensitivity in the exponent upon changes in the temperature parameter, and hence they correspond to less pronounced inflection points. Therefore in the specific case analyzed in the present paper, we rely upon our theory, as an alternative to NCCB, which does not predict a power-law behavior above the inflection temperature $T^{*}$.

Finally, we have also shown that our framework is consistent with the experimental results reported in Ref. 29 about the magnetic-field effects in the transport properties. Using a tight-binding approximation adapted to include the action of a transverse magnetic field, we have computed the change in the band structure of the thick shells in multiwalled nanotubes. In this case, the radius of the shells becomes comparable to the magnetic length, already for a magnetic field of $4 \mathrm{~T}$. Then, we have seen that the low-energy subbands show an incipient tendency to flatten along Landau levels. This may induce in general further modulation of the exponent $\alpha$, depending on the strength of the magnetic field.

We have seen that, for high values of $\alpha$ at $B=0$ (about $\approx 0.3$ ), our approach predicts a sizeable reduction of the exponent, which is in agreement with the experimental observation. As long as the density of states at the Fermi level is reduced upon application of the magnetic field, we find a picture that is consistent with the statement that, in most cases, $\alpha$ is smaller for higher magnetic fields. ${ }^{29}$ However, a clarification from the experimental point of view is required, in order to know exceptions to the rule. In our framework, we may envisage some instances in which the application of the magnetic field may induce the proximity of the Fermi level to the top of a given subband, with the consequent increase in the density of states (and the effective electronelectron interaction). We find in this way that sufficiently 
large variations in the magnetic field may produce a modulation in the exponent $\alpha$, rather than a monotonous trend. Our model provides anyhow definite predictions that are susceptible of being confronted experimentally. In this respect, more experimental input would be desirable to discern the effects of a transverse magnetic field, that may be quite significant for the outer shells in multiwalled nanotubes.

\section{ACKNOWLEDGMENTS}

J.G. acknowledges the financial support of the Ministerio de Educación y Ciencia (Spain) through Grant No. BFM2003-05317. E.P. was also supported by INFN Grant No. 10068.
${ }^{1}$ S. Iijima, Nature (London) 354, 56 (1991).

${ }^{2}$ J. W. Mintmire, B. I. Dunlap, and C. T. White, Phys. Rev. Lett. 68, 631 (1992); N. Hamada, S. I. Sawada, and A. Oshiyama, ibid. 68, 1579 (1992); R. Saito, M. Fujita, G. Dresselhaus, and M. S. Dresselhaus, Appl. Phys. Lett. 60, 2204 (1992).

${ }^{3}$ S. Frank, P. Poncharal, Z. L. Wang, and W. A. de Heer, Science 280, 1744 (1998).

${ }^{4}$ A. Urbina, I. Echeverria, A. Perez-Garrido, A. Diaz-Sanchez, and J. Abellan, Phys. Rev. Lett. 90, 106603 (2003).

${ }^{5}$ C. Schönenberger, A. Bachtold, C. Strunk, and J. P. Salvetat, Appl. Phys. A: Mater. Sci. Process. 69, 283 (1999).

${ }^{6}$ A. Bachtold, M. de Jonge, K. Grove-Rasmussen, P. L. McEuen, M. Buitelaar, and C. Schönenberger, Phys. Rev. Lett. 87, 166801 (2001).

${ }^{7}$ A. Bachtold, C. Strunk, J. P. Salvetat, J. M. Bonard, L. Forró, T. Nussbaumer, and C. Schönenberger, Nature (London) 397, 673 (1999).

${ }^{8}$ A. Kanda and Y. Ootuka, Microelectron. Eng. 63, 313 (2002).

${ }^{9}$ N. Kang, J. S. Hu, W. J. Kong, L. Lu, D. L. Zhang, Z. W. Pan, and S. S. Xie, Phys. Rev. B 66, 241403(R), (2002).

${ }^{10}$ M. Bockrath, D. H. Cobden, P. L. McEuen, N. G. Chopra, A. Zettl, A. Thess, and R. E. Smalley, Science 275, 1922 (1997).

${ }^{11}$ S. J. Tans, M. H. Devoret, R. J. A. Groeneveld, and C. Dekker, Nature (London) 386, 474 (1997).

${ }^{12}$ D. H. Cobden and J. Nygard, Phys. Rev. Lett. 89, 046803 (2002).

${ }^{13}$ W. Liang, M. Bockrath, and H. Park, Phys. Rev. Lett. 88, 126801 (2002).

${ }^{14}$ S. Bellucci and P. Onorato, Phys. Rev. B 71, 075418 (2005).

${ }^{15}$ M. R. Buitelaar, A. Bachtold, T. Nussbaumer, M. Iqbal, and C. Schönenberger, Phys. Rev. Lett. 88, 156801 (2002).

${ }^{16}$ S. Tomonaga, Prog. Theor. Phys. 5, 544 (1950); J. M. Luttinger, J. Math. Phys. 4, 1154 (1963); D. C. Mattis and E. H. Lieb, J. Math. Phys. 6, 304 (1965).

${ }^{17}$ V. J. Emery, in Highly Conducting One-Dimensional Solids, edited by J. T. Devreese, R. P. Evrard, and V. E. Van Doren (Plenum, New York, 1979).

${ }^{18}$ J. Solyom, Adv. Phys. 28, 201 (1979).

${ }^{19}$ F. D. M. Haldane, J. Phys. C 14, 2585 (1981).

${ }^{20}$ M. Bockrath, D. H. Cobden, J. Lu, A. G. Rinzler, G. Andrew, R. E. Smalley, L. Balents, and P. L. McEuen, Nature (London) 397, 598 (1999).

${ }^{21}$ J. Nygard, D. H. Cobden, M. Bockrath, P. L. McEuen, and P. E. Lindelof, Appl. Phys. A 69, 297 (1999).

${ }^{22}$ Z. Yao, H. W. J. Postma, L. Balents, and C. Dekker, Nature (London) 402, 273 (1999).
${ }^{23}$ H. W. C. Postma, M. de Jonge, Z. Yao, and C. Dekker, Phys. Rev. B 62, R10653 (2000); H. W. C. Postma, T. Teepen, Z. Yao, M. Grifoni, and C. Dekker, Science 293, 76 (2001).

${ }^{24}$ C. L. Kane and M. P. A. Fisher, Phys. Rev. B 46, 15233 (1992); Phys. Rev. Lett. 68, 1220 (1992).

${ }^{25}$ S. Bellucci and J. González, Eur. Phys. J. B 18, 3 (2000); S. Bellucci, Path Integrals from peV to TeV, edited by R. Casalbuoni, R. Giachetti, V. Tognetti, R. Vaia, and P. Verrucchi (World Scientific, Singapore, 1999), p. 363.

${ }^{26}$ S. Bellucci and J. González, Phys. Rev. B 64, 201106(R) (2001).

${ }^{27}$ S. Bellucci, J. González, and P. Onorato, Nucl. Phys. B 663, 605 (2003).

${ }^{28}$ S. Bellucci, J. González, and P. Onorato, Phys. Rev. B 69, 085404 (2004).

${ }^{29}$ A. Kanda, K. Tsukagoshi, Y. Aoyagi, and Y. Ootuka, Phys. Rev. Lett. 92, 036801 (2004).

${ }^{30}$ R. Egger, Phys. Rev. Lett. 83, 5547 (1999).

${ }^{31}$ R. Egger and A. O. Gogolin, Phys. Rev. Lett. 87, 066401 (2001).

${ }^{32}$ H. J. Choi, J. Ihm, S. G. Louie, and M. L. Cohen, Phys. Rev. Lett. 84, 2917 (2000).

${ }^{33}$ S. Bellucci, J. González, and P. Onorato, Phys. Rev. Lett. 95, 186403 (2005).

${ }^{34}$ C. W. J. Beenakker, Phys. Rev. B 44, 1646 (1991).

${ }^{35}$ R. Egger and A. O. Gogolin, Phys. Rev. Lett. 79, 5082 (1997); Eur. Phys. J. B 3, 281 (1998).

${ }^{36}$ C. Kane, L. Balents, and M. P. A. Fisher, Phys. Rev. Lett. 79, 5086 (1997).

${ }^{37}$ R. Egger and H. Grabert, Phys. Rev. Lett. 79, 3463 (1997).

${ }^{38}$ For the dominant forward-scattering processes considered here, a sensible average of the interaction is given by $q_{0} \sim 1 / L$.

${ }^{39}$ M. Krüger, M. R. Buitelaar, T. Nussbaumer, C. Schönenberger, and L. Forró, Appl. Phys. Lett. 78, 1291 (2001).

${ }^{40}$ R. Saito, G. Dresselhaus, and M. S. Dresselhaus, Physical Properties of Carbon Nanotubes (Imperial College Press, London, 1998), Chap. 6.

${ }^{41}$ H.-W. Lee and D. S. Novikov, Phys. Rev. B 68, 155402 (2003).

${ }^{42}$ One cannot discard, however, a nontrivial interplay between the quantum Hall regime and the Luttinger liquid regime at large magnetic fields. Perturbative evidence of that has been provided, for instance, in Ref. 43.

${ }^{43}$ S. Bellucci and P. Onorato, Ann. Phys. (N.Y.) 321, 934 (2006); S. Bellucci and P. Onorato, Eur. Phys. J. B 45, 87 (2005).

${ }^{44}$ T. Kanbara, T. Iwasa, K. Tsukagoshi, Y. Aoyagi, and Y. Iwasa, Appl. Phys. Lett. 85, 6404 (2004). 